\title{
Panhipopituitarismo secundario a metástasis del sistema nervioso central por un adenocarcinoma broncogénico
}

\section{Panhypopituitarism secondary to metastasis of the central nervous system due to a bronchogenic adenocarcinoma}

\author{
Jovani Osorno, Manuel Felipe Cáceres, Juan David Cáceres, \\ Jaime Andrés Calle, Miguel Ignacio Roldán, Juan Paulo Sandoval \\ - Medellín (Colombia)
}

\section{Resumen}

Mujer de 45 años de edad con antecedente de extabaquismo pesado, cursó con cuadro crónico de cefalea en región occipital irradiado a región temporal asociado a disminución de la agudeza visual de ojo izquierdo. El cuadro empeoró en los últimos seis meses y se asoció a polidipsia y poliuria. Presentó cuadro sincopal con amnesia retrógrada, para lo cual se realizó una tomografía axial computarizada (TAC) contrastada de cráneo que mostró múltiples lesiones parenquimatosas cerebrales y del cerebelo compatibles con metástasis. Se hizo estudio hormonal para evaluar la función hipofisiaria evidenciando un panhipopituitarismo secundario. Se detectó foco primario neoplásico mediante TAC contrastada de tórax, evidenciando una lesión espiculada en el lóbulo superior derecho sugestiva de carcinoma broncogénico, posteriormente se tomó biopsia por fibrobroncoscopia el cual confirmó por histopatología e inmunohistoquímica el diagnóstico de un adenocarcinoma broncogénico. (Acta Med Colomb 2018; 43: 115-118).

Palabras clave: hipopituitarismo, carcinoma broncogénico, metástasis de la neoplasia.

\section{Abstract}

A 45-year-old woman with a history of heavy extabaquism presented with chronic headache in the occipital region irradiated to the temporal region associated with decreased visual acuity of the left eye. The picture worsened in the last six months and was associated with polydipsia and polyuria. She presented a syncopal picture with retrograde amnesia, for which a contrast computed tomography (CT) of the skull was performed, which showed multiple parenchymal brain and cerebellar lesions compatible with metastasis. Hormonal study was done to evaluate the hypophyseal function evidencing a secondary panhypopituitarism. A primary neoplastic focus was detected by a contrast chest CT scan, showing a spiculated lesion in the right upper lobe suggestive of bronchogenic carcinoma. A biopsy was subsequently taken by fibrobronchoscopy, which confirmed the diagnosis of a bronchogenic adenocarcinoma by histopathology and immunohistochemistry. (Acta Med Colomb 2018; 43: 115-118).

Keywords: hypopituitarism; bronchogenic carcinoma; metastasis of the neoplasia
Dr. Jovani Osorno Serna: Especialista en Medicina Interna, Neumología, Oncología Torácica y Epidemiología. Docente del Departamento de Medicina Interna. Hospital Universitario San Vicente de Paúl Fundación; Dr. Manuel Felipe Cáceres Acosta: Residente de Medicina Interna, Universidad del Cauca; Juan David Cáceres González: Estudiante de Pregrado de Medicina, Universidad de Antioquia, Dr. Jaime Andrés Calle Ramírez: Residente de Medicina Interna, Universidad de Antioquia; Dr. Miguel Ignacio Roldán Pérez: Especialista en Patología. Docente del Departamento de Patología, Hospital Universitario San Vicente de Paúl Fundación, Dr. Juan Paulo Sandoval Mesa: Residente de Patología, Universidad de Antioquia. Medellín (Colombia).

Correspondencia: Dr. Jovani Osorno Serna. Medellín (Colombia).

E-mail: giosorno@gmail.com

Recibido: 25/VI/2017 Aceptado: 23/IV/2018

\section{Introducción}

Las metástasis hipofisarias ocurren alrededor de 1\% de los pacientes con tumores malignos y son una causa rara de panhipopituitarismo (1). Tenemos un caso de una rara presentación de panhipopituitarismo secundario a una metástasis hipofisiaria debido a un adenocarcinoma broncogénico.

\section{Presentación del caso}

Mujer de 45 años de edad, índice de Karnofsky de 70\%, exfumadora pesada con índice de 25 años/paquete que suspendió hace un año, sin exposición al humo de leña, con antecedentes de radiculopatía en raíces nerviosas en L4-L5S1 que requirió bloqueo selectivo de L4-L5, tubectomía hace 
varios años, gestaciones seis, partos seis, abortos cero; última citología de cérvix uterino de hace dos años sin anormalidades, con irregularidades menstruales desde hace tres meses, antes tenía ciclos regulares $28 / 5$. Sus antecedentes familiares eran hipertensión arterial y diabetes mellitus tipo 2 .

Consultó a una institución de cuarto nivel de Medellín por cuadro de un año de evolución, caracterizado por cefalea en región occipital irradiado a región frontal asociado a disminución de la agudeza visual en el ojo izquierdo, ambos sin mejoría a pesar de manejo médico. En los últimos seis meses la sintomatología empeoró al presentar cefalea intensa en la escala análoga del dolor, pérdida de la agudeza visual del ojo izquierdo, polidipsia y poliuria. En los últimos tres días el dolor se hizo insoportable y presentó posteriormente episodio sincopal con pérdida de la conciencia de dos minutos y amnesia retrógrada, sin movimientos anormales ni pérdida del control de esfínteres, negó tos, disnea o hemoptisis.

Al examen físico se encontró alerta, orientada en tiempo, espacio y persona, estable hemodinamicamente, afebril, álgica, con mucosas secas, sin asimetría facial, sensibilidad en cara conservada, movimientos de la lengua conservados, anisocoria, defecto pupilar aferente bilateral, ojo izquierdo solo percibía la luz, ojo derecho alcanzaba a contar dedos a $30 \mathrm{~cm}$, sin oculoparesias, examen oftalmológico normal incluyendo el disco óptico, fuerza segmentaria conservada, sin paresias, reflejos osteotendinosos con hiperreflexia en miembro inferior izquierdo y normalidad en miembro superior ipsilateral, resto de reflejos normales, respuesta plantar neutra bilateral. Se abordó el cuadro como una cefalea crónica con banderas rojas siendo necesaria una tomografía axial computarizada (TAC) de cráneo simple, en donde se encontraron tres lesiones redondeadas, bien delimitadas, una de localización frontal derecha de menos de $1 \mathrm{~cm}$ de diámetro con edema vasogénico perilesional, otra localizada en el lóbulo parietal ipsilateral con características similares a la lesión frontal y una lesión selar de $15 \mathrm{~mm}$ de longitud siendo necesario caracterizar mejor las lesiones con estudio contrastado (Figura 1), el cual mostró múltiples lesiones parenquimatosas cerebrales, hipervascularizadas que captaban intensamente el contraste, con edema vasogénico en lóbulos frontal y parietal derechos, región hipofisiaria y cerebelosa derecha compatibles con metástasis. Algunas lesiones con extensión supraselar que desplazaba el quiasma óptico hacia superior. Se decidió iniciar manejo profiláctico anticonvulsivante con fenitoína sódica $100 \mathrm{mg}$ cada 8 horas y manejo del edema cerebral con dexametasona $4 \mathrm{mg}$ intravenosa cada 8 horas previa desparasitación.

Debido al compromiso hipofisiario, se realizó perfil hormonal que arrojó los resultados mostrados en la Tabla 1 , con una función renal y electrólitos (incluyendo magnesio y calcio) dentro de parámetros de normalidad. Ante la sos-

Tabla 1. Perfil hormonal investigando alteración hipofisiaria.

\begin{tabular}{|l|c|c|}
\hline & Valor & Rango de referencia \\
\hline T4 libre & $8.36 \mathrm{pmol} / \mathrm{L}$ & $0.9-19 \mathrm{pmol} / \mathrm{L}$ \\
\hline TSH & $2.38 \mathrm{mU} / \mathrm{L}$ & $0.35-4.94 \mathrm{mU} / \mathrm{L}$ \\
\hline Cortisol & $16.5 \mathrm{nmol} / \mathrm{L}$ & $80-477 \mathrm{nmol} / \mathrm{L}$ \\
\hline Prolactina & $67.3 \mu \mathrm{g} / \mathrm{L}$ & $5.18-26.53 \mu \mathrm{g} / \mathrm{L}$ \\
\hline LH & $0 \mathrm{IU} / \mathrm{L}$ & $0.4-20 \mathrm{IU} / \mathrm{L}$ \\
\hline FSH & $0.3 \mathrm{IU} / \mathrm{L}$ & $1.38-5.47 \mathrm{IU} / \mathrm{L}$ \\
\hline HbA1c & $5.50 \%$ & $3-6.5 \%$ \\
\hline Osmolaridad urinaria & $251 \mathrm{mmol} / \mathrm{Kg}$ & $392-1092 \mathrm{mmol} / \mathrm{Kg}$ \\
\hline $\begin{array}{l}\text { TSH: hormona estimulante de la tiroides; } \mathrm{LH}: \text { hormona luteinizante; } \mathrm{FSH}: \text { hormona } \\
\text { foliculoestimulante; HbA1c: hemoglobina glicada. }\end{array}$
\end{tabular}

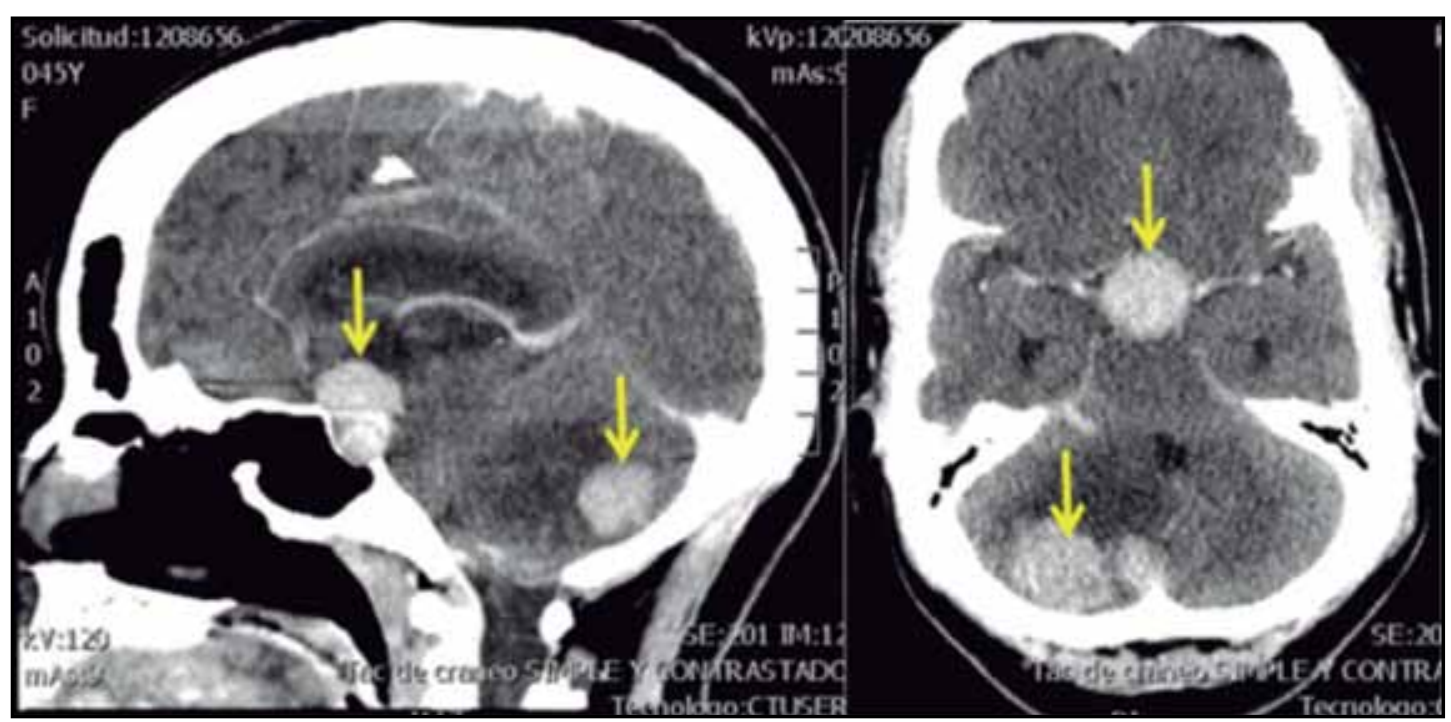

Figura 1. TAC contrastada de cráneo corte sagital y axial. Se observan múltiples lesiones nodulares de comportamiento hipervascular posterior a la administración del medio de contraste localizadas en el hemisferio cerebeloso derecho y la región hipofisiaria (flechas). Las lesiones índice localizadas en el hemisferio cerebeloso derecho de $32 \mathrm{~mm}$ y en la región hipofisiaria de $21 \mathrm{~mm}$ con similar densidad, lo que sugiere lesiones metastásicas. 
pecha de diabetes insípida se realizó test de desmopresina evaluando la osmolaridad urinaria basal (Tabla 1) y 12 horas posteriores a su administración, la cual fue de 734 mmol/Kg (392 - 1092 mmol/Kg). Determinándose con los hallazgos de laboratorio e imagenológicos un panhipopituitarismo (hipotiroidismo central, hipogonadismo central, hipocortisolismo a pesar de suplencia con dexametasona de manera intrahospitalaria y diabetes insípida) asociado a hiperprolactinemia compresiva. Se le inicio suplencia con levotiroxina $75 \mu \mathrm{g} /$ día y desmopresina $10 \mu \mathrm{g} 1$ puff nasal cada 12 horas.

Ante los hallazgos identificados en las imágenes previas, se hicieron estudios de extensión para identificar foco neoplásico primario, la TAC contrastada de abdomen no mostró lesiones sugestivas de primario y la TAC contrastada de tórax (Figura 2) evidenció una masa pulmonar espiculada sin cavitación en el lóbulo superior derecho, adenopatías de

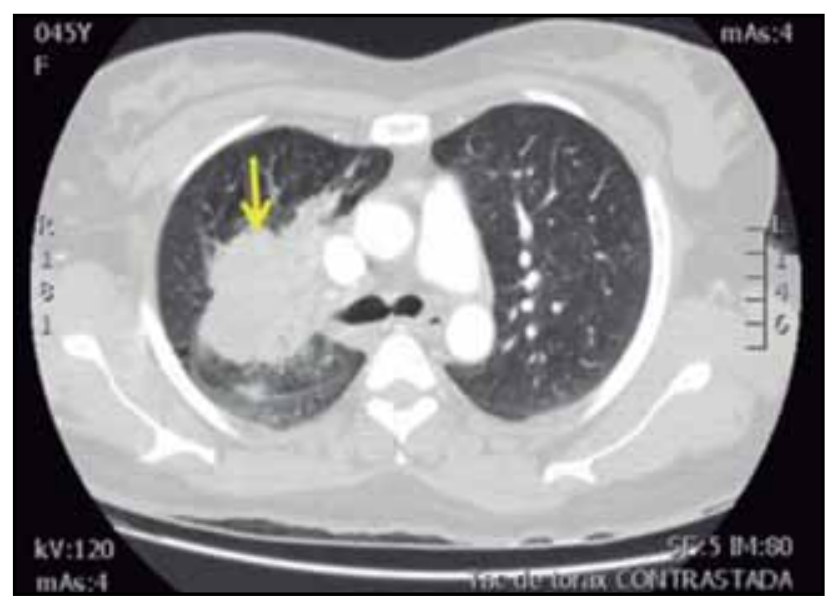

Figura 2. TAC contrastada de tórax corte axial. Se observa masa de contornos espiculados (flecha), con densidad de tejidos blandos localizada en el lóbulo superior derecho comprometiendo el bronquio para el lóbulo superior, con unas dimensiones de $67 \times 57 \times 57 \mathrm{~mm}$, sin evidencia de cavitación central.

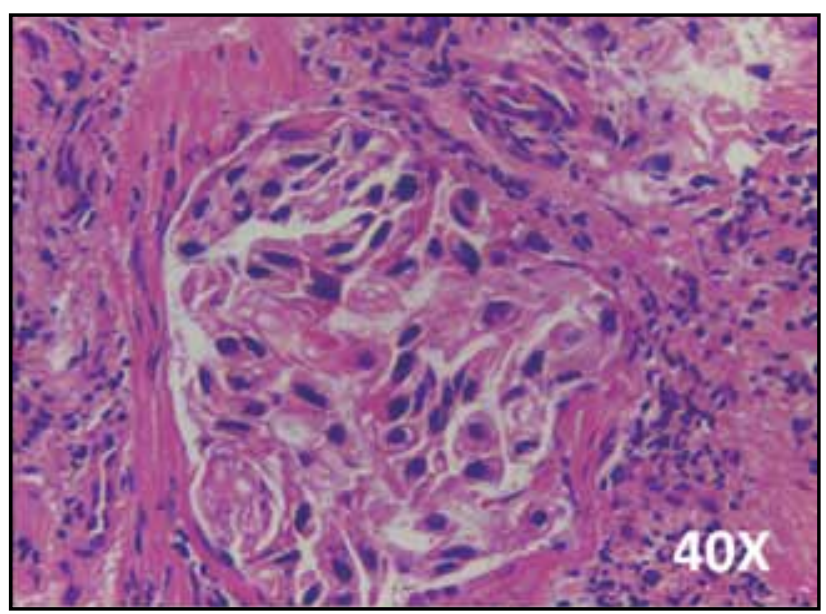

Figura 3. Tinción hematoxilina-eosina de biopsia pulmonar. Técnica microscopía de luz. Coloración hematoxilina-eosina. Aumento 40x. Se observa infiltración por estructuras neoplásicas de aspecto glandular tapizadas por células de núcleos pleomórficos, algunas con nucléolos de citoplasma amplio eosinófilo. Los hallazgos son compatibles morfológicamente con un adenocarcinoma broncogénico. localización hiliar derecha de $15 \mathrm{~mm}$ de diámetro en el eje corto y paratraqueales derechas de $13 \mathrm{~mm}$ de diámetro en el eje corto, sin lesiones líticas o blásticas en las estructuras óseas. La lesión pulmonar era sugestiva de carcinoma broncogénico siendo necesaria su confirmación histológica por biopsia mediante fibrobroncoscopia. Los hallazgos de la biopsia confirmaron el diagnóstico de un carcinoma de célula no pequeña compatible morfológicamente con un adenocarcinoma broncogénico (Figura 3), los marcadores de inmunohistoquímica fueron positivo para TTF1 (Figura 4) y negativo para P63 (Figura 5).

Debido a lo avanzado de su enfermedad, se clasificó en un estadio IV, por lo se le decidió iniciar teleterapia holoencefálica paliativa. Posteriormente se le hizo gammagrafía ósea con tecnecio-99m, la cual descartó metástasis en otros sitios. Durante evolución presentó convulsiones las cuales se manejaron por el grupo de neurología adicionándole

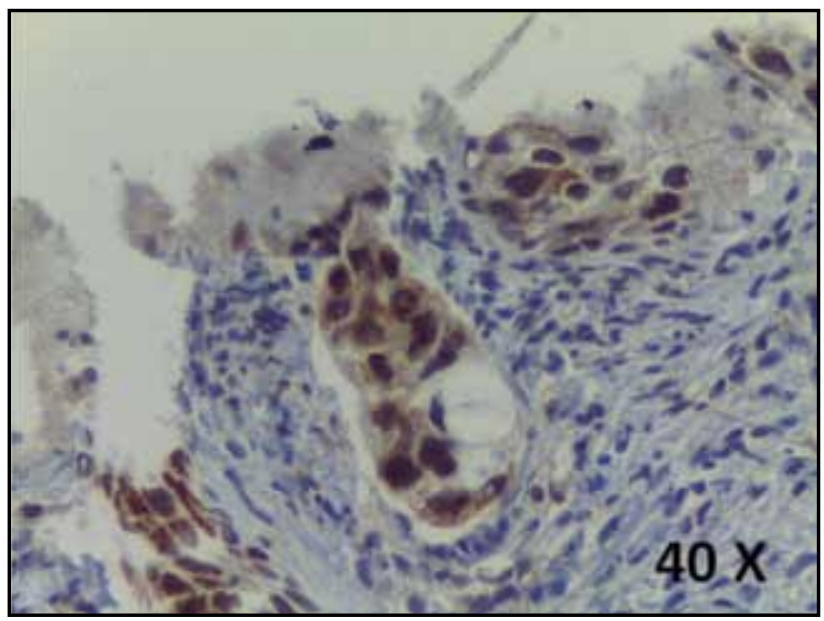

Figura 4. Marcadores para inmunohistoquímica para TTF-1 de biopsia pulmonar. Técnica microscopía de luz. Inmunohistoquímica polímero-peroxidasa. Aumento 40x.TTF-1 positiva nuclear en las estructuras glandulares neoplásicas.

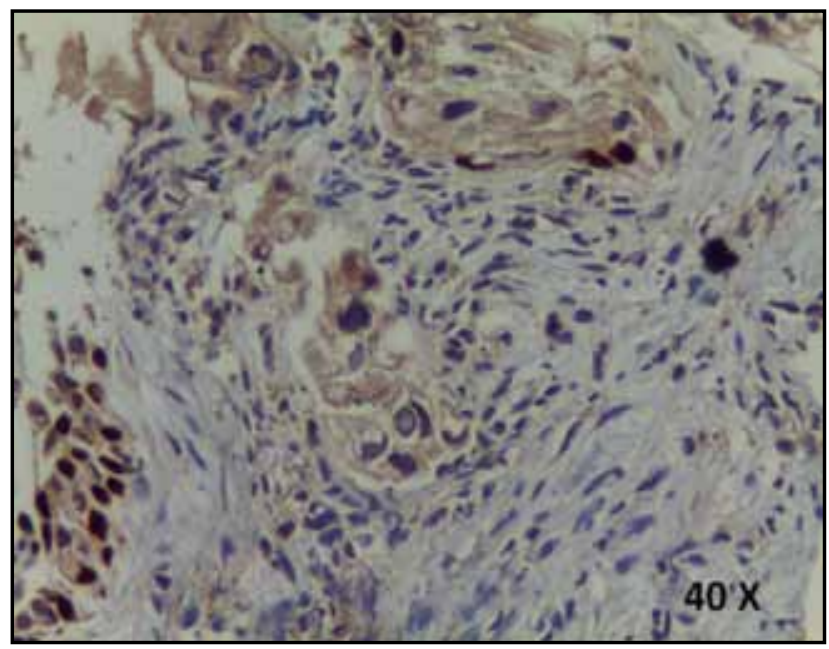

Figura 5. Marcadores para inmunohistoquímica para P63 de biopsia pulmonar. Técnica microscopía de luz. Inmunohistoquímica polímero-peroxidasa. Aumento 40x. P63 negativa nuclear en las estructuras glandulares neoplásicas. 
levetiracetam, con adecuado control. Egresó para continuar manejo por cuidados paliativos y oncología clínica.

\section{Discusión}

La metástasis a nivel hipofisiario no son comunes, ocurren alrededor de $1 \%$ de los pacientes con tumores malignos y son una causa rara de panhipopituitarismo. Las primeras descripciones sobre disfunción hipofisiaria asociada a carcinoma broncogénico fueron realizadas entre los años 50 y 70 $(2,3)$, en estos informes no se obtuvo datos claros de un hipopituitarismo clínico evidente (4). En una serie de autopsias de Teears et al, sólo 6.8\% de las metástasis hipofisarias presentaron clínica de disfunción de hipofisiaria, con afectación más frecuente del lóbulo posterior (56.8\%) y la localización del tumor primario fue mama y pulmón (mujer y hombre respectivamente) (5). La mayoría de anormalidades endocrinológicas asociadas con el carcinoma broncogénico resulta de una excesiva secreción de hormonas o sustancias similares a las hormonas como la secreción inadecuada de $\mathrm{ADH}$ o el síndrome de Cushing. El panhipopituitarismo es inusual y la sintomatología que se presenta con más frecuencia es la diabetes insípida y de forma infrecuente la afectación del lóbulo anterior hipofisiario (6), en el caso de la paciente se evidenció un hipotiroidismo central, hipogonadismo central, hipocortisolismo, diabetes insípida (confirmada por el test de desmopresina y medición de osmolaridad urinaria), además hiperprolactinemia por efecto compresivo metastásico, aunque se resalta que hay reportes de prolactinoma asociado (7). El clínico debe sospechar metástasis hipofisiaria con la siguiente triada clínica: diabetes insípida (polidipsia, poliuria y sed), oftalmoplejía y cefalea en un paciente mayor de 50 años, independientemente de una historia de malignidad (8-10), en ocasiones, la manifestación de convulsiones y cefalea puede sugerir compromiso hipofisiario (11). Es de resaltar que no se realizó una resonancia nuclear magnética (RMN) de hipófisis en esta paciente, debido a que las densidades de las metástasis en el TAC contrastado de cráneo mostraban un valor semejante, además la evidencia clínica de la diabetes insípida sólo se presenta en 3\% de los casos de adenoma (12). El tratamiento en estos casos es paliativo y se enfoca principalmente en mejorar la calidad de vida del paciente, se utilizan la sustitución hormonal, radioterapia y quimioterapia (13). Es infrecuente que se presente metástasis hipofisiario de un adenocarcinoma broncogénico (14), ya que el más frecuente es el de células pequeñas (15), en el caso de la paciente se presentó este raro hallazgo.

\section{Agradecimientos}

Al convenio entre la Universidad del Cauca y la Universidad de Antioquia por enriquecer la producción académica.

\section{Referencias}

1. Guijarro de Armas MG, Torán Ranero CE, Pavón de Paz I, Merino Viveros M, Iglesias Bolaños P, Monereo Megías S. Panhypopituitarism and lung neoplasm: a case study. Endocrinol Nutr. 2013 Dec; 60 (10): e35-6.

2. Peabody HD Jr, Olson AM. Association of bronchogenic carcinoma and diabetes insipidus: Report of cases. Proc Staff Meet Mayo Clin. 1951; (26): 107.

3. Gailani SD, Roque AL, Band P, Ross C. Hypopituitarism due to localized hypothalamic lesioons. Arch Intern Med. 1970 Aug; 126 (2): 284-286.

4. Epstein S, Ranchod M, Goldswain PR. Pituitary insufficiency, inappropriate antidiuretic hormone (ADH) secretion, and carcinoma of the bronchus. Cancer. 1973 Aug; 32 (2): 476-481.

5. Teears RJ, Silverman EM. Clinicopathologic review of 88 cases of carcinoma metastatic to the pituitary gland. Cancer. 1975; 36 (1): 216-220.

6. Ko JC, Yang PC, Huang TS, Yeh KH, Kuo SH, Luh KT. Panhypopituitarism caused by solitary parasellar metastasis from lung cancer. Chest. 1994 Mar; 105 (3): 951-953.

7. Siqueira PF de, Mathez ALG, Pedretti DB, Abucham J. Pituitary metastasis of lung neuroendocrine carcinoma: case report and literature review. Arch Endocrinol Metab. 2015 Dec; 59 (6): 548-53.

8. Komninos J, Vlassopoulou V, Protopapa D, et al. Tumors metastatic to the pituitary gland: Case report and literature review. J Clin Endocrinol Metab. 2004; (89): 574-80.

9. Branch Jr CL, Laws Jr ER. Metastatic tumors of the sella turcica masquerading as primary pituitary tumors. J Clin Endocrinol Metab. 1987; (65): 469-74.

10. He W, Chen F, Dalm B, Kirby PA, Greenlee JD. Metastatic involvement of the pituitary gland: A systematic review with pooled individual patient data analysis. Pituitary. 2015; (18): 159-68.

11. Fridley J, Adams G, Rao V, Patel A, Humphries W, Goodman C, et al. Small cell lung cancer metastasis in the pituitary gland presenting with seizures and headache. J Clin Neurosci. 2011 Mar; 18 (3): 420-2.

12. Nattero L, Martínez M, Santana B, Luque-Ramírez L. Symptomatic metastasis of the pituitary stem secondary to disseminated pulmonary neoplastic disease. Rev Clin Esp. 2009; (210): 595-6.

13. Johnston PC, Black NR, McIlroy J, Sharkey R, Garvey A, Lindsay JR. Hypopituitarism as the presenting feature of bronchogenic carcinoma with metastases to the pituitary gland. Indian J Endocrinol Metab. 2013 Jan; 17 (1): 167-9.

14. Gulati S, Kiefer C, Karim NA. Diabetes Insipidus: An Unusual Presentation of Adenocarcinoma of the Lung in a Patient with no Identifiable Lung Mass. $N$ Am J Med Sci. 2015 Oct; 7 (10): 476-9.

15. Pérez-Ferrer $P$, Landete $P$, Chiner $\mathbf{E}$. Hipopituitarismo como forma de inicio de un carcinoma microcítico de pulmón. Archi Bronconeumo. 2013; 49 (5): 216-7. 